\title{
HYBRID TOTAL HIP REPLACEMENT
}

\author{
A 6.5-YEAR FOLLOW-UP STUDY
}

THOMAS P. SCHMALZRIED, WILLIAM H. HARRIS

From Massachusetts General Hospital and Harvard Medical School, Boston, USA

\begin{abstract}
We have reviewed 97 consecutive primary hip replacements with a cemented femoral component and a porous-ingrowth acetabular component at a minimum five-year follow-up (average 6.5 years). The average Harris hip score was 93, and 85 hips had no pain or only slight pain. There had been no deterioration in the results since the two-year follow-up.

The hybrid hip is successful for up to eight years and appears to be suitable for many patients. Long-term femoral fixation has been shown to improve with secondgeneration cementing techniques and in this series was excellent with third-generation techniques, in that only one stem was revised for loosening. No cementless acetabular component was revised for loosening.
\end{abstract}

J Bone Joint Surg [Br] 1993; 75-B :608-15.

Received 18 June 1992; Accepted after revision 3 December 1992

The hybrid total hip replacement combines a cemented femoral component with a cementless acetabular component (Davey and Harris 1989). We found excellent clinical and radiographic results at a minimum two-year follow-up of 126 consecutive primary hybrid replacements (Harris and Maloney 1989). We now report the clinical and radiographic results of the subset of this same cohort of patients which has reached a minimum five-year follow-up.

\section{PATIENTS AND METHODS}

Between November 1982 and December 1985, 101 consecutive primary hybrid total hip arthroplasties were performed in 95 patients by the senior author. In the first 52 hips, the cemented femoral component was either an HD-2 (Howmedica Inc, Rutherford, New Jersey) cobaltchrome implant with a medial collar (50 hips) or (in two

T. P. Schmalzried, MD, Assistant Professor of Orthopaedic Surgery Harbor-UCLA Medical Center and Joint Replacement Institute, 1000 W. Carson Street, Los Angeles, California 90509, USA.

W. H. Harris, MD, Chief, Hip and Implant Unit

Orthopaedic Biomechanics Laboratory, Massachusetts General Hospital, 32 Fruit Street, Boston, Massachusetts 02114, USA.

Correspondence should be sent to Dr W. H. Harris.

(C) 1993 British Editorial Society of Bone and Joint Surgery 0301-620X/93/4586 \$2.00 hips) a custom $\mathrm{CDH}$ component (Zimmer Inc, Warsaw, Indiana). The acetabular implant was hemispherical, of cobalt-chrome with sintered cobalt-chrome beads at the bone-implant interface, called the acetabular reconstruction component (ARC; Howmedica). Initial fixation was by screws, roughly parallel to each other, placed in three peripheral flanges which projected from the rim of the component (Fig. 1).

In the other 49 hips, a Precoat (Zimmer) femoral stem was cemented in place in 42 and a custom $C D H$ Precoat (Zimmer) stem was used in the remaining seven. All components had collars and were made of forged cobalt-chrome. The hemispherical acetabular component was of titanium with sintered titanium fibre mesh (HarrisGalante porous, HGP; Zimmer), and had multiple holes for screw fixation through the shell (Fig. 2). The polyethylene liner was held in place by titanium tines around the mouth of the shell.

Most of the femoral heads were $26 \mathrm{~mm}$ in diameter and the maximum diameter used was $28 \mathrm{~mm}$. The femoral head was integral in the HD-2 prostheses, but modular in the Precoat prostheses.

There were 91 surviving patients (96 hips) all assessed clinically. Radiographs were not obtained for eight hips (3 ARC, 5 HGP), all of which were functioning well with a mean Harris hip score of 92. None had reoperation or revision. Current radiographs were available for 89 hips (48 ARC, 41 HGP).

Mean follow-up was 6.5 years ( 5 to 8 ). There were 48 women and 43 men; their average age was 61 years (23 to 83). The diagnosis was osteoarthritis in 68 hips, avascular necrosis in 12, congenital dislocation in 10 , rheumatoid arthritis in 5 , nonunion of a subcapital fracture in one, and multiple epiphyseal dysplasia in one. Surgical techniques. The femoral canal is rasped then irrigated by a pulsatile lavage system. A special instrument, the calcar planer, is used to prepare the medial femoral neck to allow exact collar-calcar contact (see Figs 1 and 5a). The femoral canal is plugged with methylmethacrylate, and haemostasis is maximised by packing the canal with a sponge soaked in dilute adrenaline solution. Simplex bone cement (Howmedica) is centrifuged to reduce porosity and improve fatigue strength (Burke, Gates and Harris 1984), then inserted in a retrograde fashion by a cement gun. A femoral seal is used to occlude the opening of the femoral neck and 


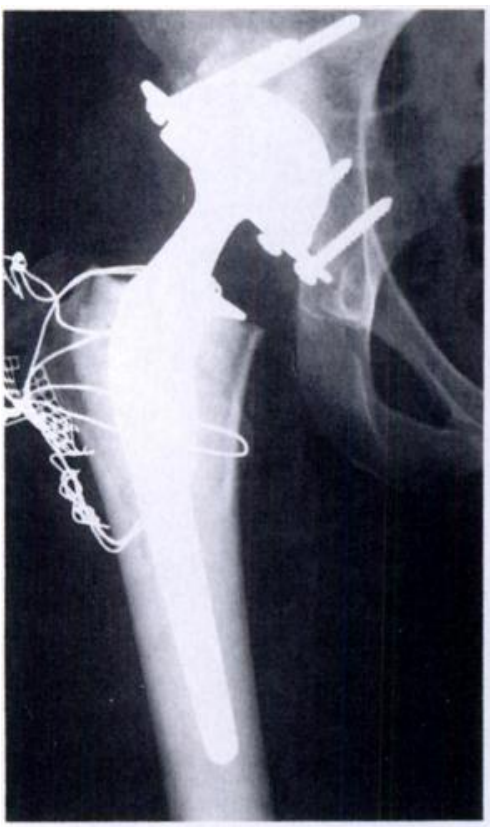

Fig. 1

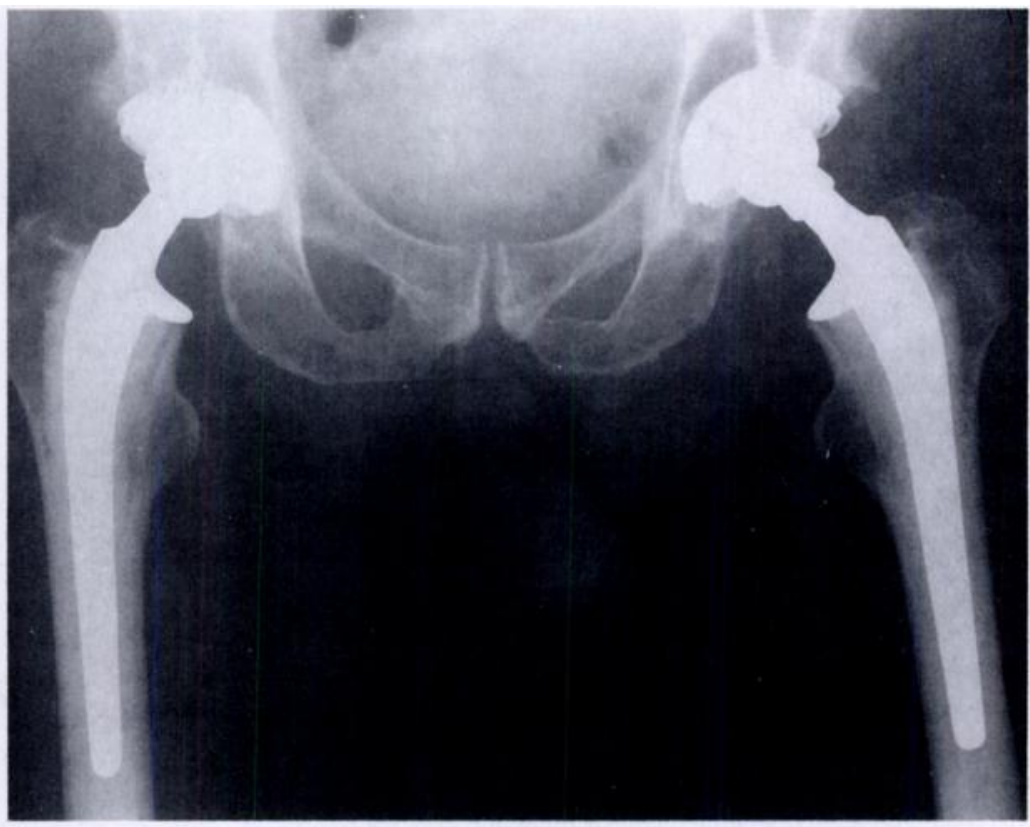

Fig. 2

Figure 1 - ARC hybrid total hip replacement. A beaded porous-surface, cobalt-chrome, ARC socket is paired with an HD-2 stem. The cement penetrates the cancellous bone to the cortices in the diaphysis, but there is a defect in the cement mantle medially (isolated femoral zone 5) where the tip of the prosthesis contacts the femoral cortex, reducing the cement mantle to grade $C$. Three screws were used to fix the ARC. The fourth screw, with a different thread, was inserted at the time of the prior acetabular fracture. Figure 2 - Bilateral HGP hybrid total hip replacements. The titanium mesh porous-surface HGP sockets are paired with Precoat femoral stems. The cement mantles are at least $2 \mathrm{~mm}$ thick, completely surrounding the stem with no focal defects, voids or bubbles and extend at least $2 \mathrm{~cm}$ below the tip of the prosthesis. Cement penetrates the cancellous bone to the cortices in the diaphysis to produce 'white-out' (grade A cementing).

allow pressurisation of the cement before the insertion of the femoral component (Oh, Bourne and Harris 1983).

We have designated the original femoral cementing technique of Charnley (finger-packing into an unplugged canal) as first-generation cementing. The use of a medullary plug and a cement gun (second-generation cementing) has improved the durability of cemented femoral fixation (Roberts, Poss and Kelley 1986; Wroblewski 1986; Russotti, Coventry and Stauffer 1988; Mulroy and Harris 1990; Barrack, Mulroy and Harris 1992). Third-generation techniques include pulsatile lavage, reduction of cement porosity, pressurisation, and precoating. All cases in this series had pulsatile lavage, porosity reduction and pressurisation. The second group (49 hips) also had precoated femoral stems.

The technique for acetabular insertion is similar for both types of component. Hemispherical reamers are used to maximise the cancellous bone surface so that the outer diameter of the porous acetabular component is equal to the outer diameter of the last reamer used. There is no 'press-fit' stability, and the component is fixed with screws.

Assessment of outcome. The Harris hip score (1969) was used to grade hip function. Current anteroposterior views of the hip and pelvis, plus frog and true lateral views of the hip, were compared with the first postoperative and two-year follow-up radiographs. All patients also had Judet views (Judet, Judet and Letournel 1964) of the pelvis postoperatively; 58 had these views at two-year follow-up and 60 at final follow-up (Kwong et al 1991; Schmalzried et al 1992b).

Radiographic evaluation of the femoral component included assessment for collar-calcar contact, stem position, cement technique, adequacy of the cement mantle, areas of bone resorption and regions of bony remodelling on both the anteroposterior and lateral views. We graded cement technique and adequacy of the cement mantle by the following new criteria:

Grade $A$ was complete filling of the cement-bone interface in the diaphyseal and metaphyseal regions (a so-called 'white-out'), with cement extending at least $2 \mathrm{~cm}$ past the tip of the prosthesis. The cement mantle was at least $2 \mathrm{~mm}$ thick, and completely surrounded the prosthesis with no focal defects (Fig. 2).

Grade $B$ required the same stringent criteria for the mantle, but cement penetration did not extend completely through the cancellous bone to the cortices to produce a white-out. In this grade, there may be areas of trabecular bone at the cement-bone interface in the diaphyseal area which are not filled.

Grade $C$ included cement mantles with focal deficiencies (Maloney et al 1989; Jasty et al 1990) such as any portion of the stem in contact with cortical bone, or radiographic voids or bubbles in the cement (Fig. 1).

Grade $D$ stems had either multiple focal and/or extensive diffuse mantle defects (Carlsson, Gentz and Linder 1983; Anthony et al 1990), no cement past the stem tip, or lucencies at the stem-cement interface. 


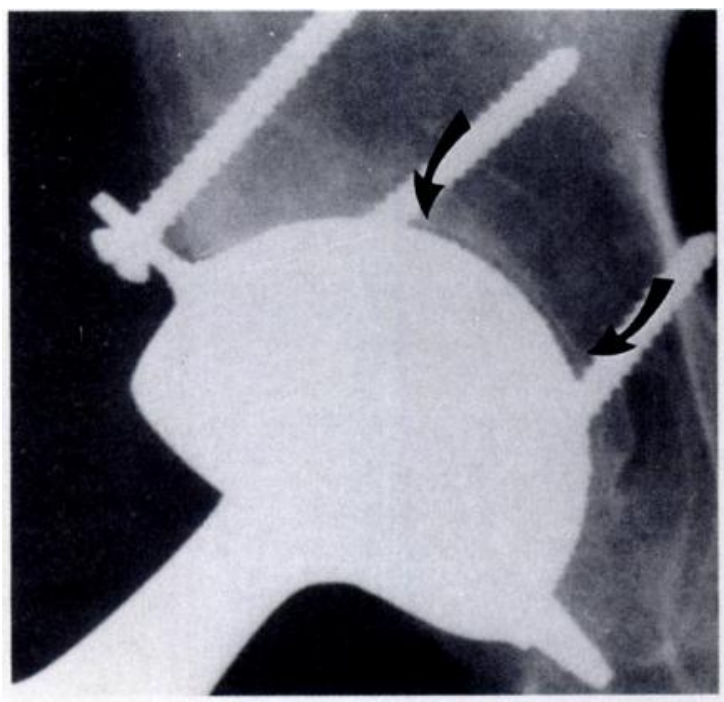

Fig. 3a

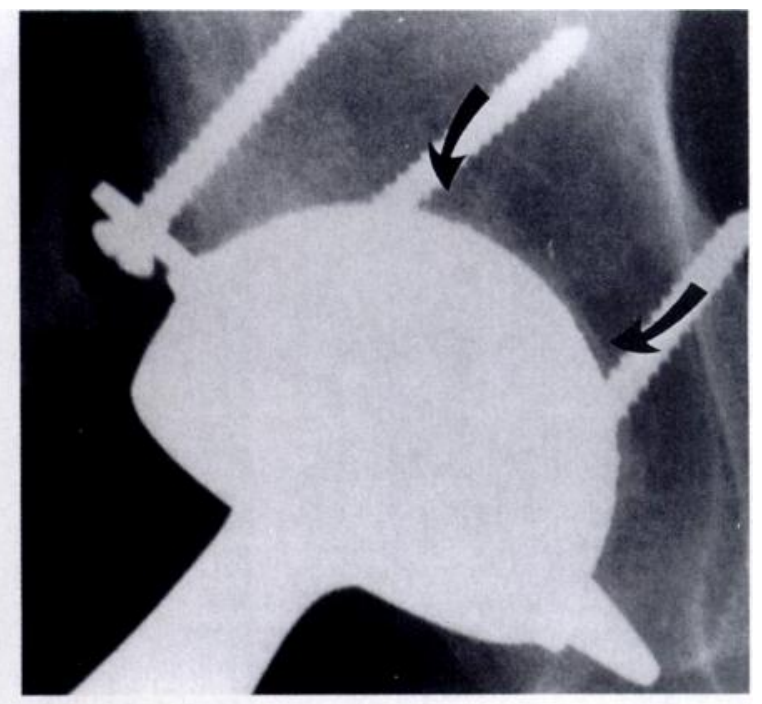

Fig. 3b

Figure 3a-Postoperative radiograph of an ARC socket; there is a $1 \mathrm{~mm}$ gap in zone 2 between the porous surface and the bone. Figure $3 b-$ On the two-year follow-up film, the gap has decreased substantially.

We compared the immediate postoperative, twoyear and final follow-up films. The location of radiographic findings around the femoral prosthesis was described using 14 zones (Johnston et al 1990). Definite loosening was diagnosed if there was subsidence of the prosthesis, any radiolucency at the metal-cement interface, a cement fracture, stem bending, or stem fracture (Harris, McCarthy and O'Neill 1982). Subsidence was evaluated by measuring both the distance from the centre of the prosthetic head and the distance from the collar to the most proximal part of the lesser trochanter.

The position of the acetabular component was assessed on the anteroposterior and true lateral radiographs, and the percentage of bone contact on the anteroposterior projection. Radiolucent lines on the acetabulum were defined as described by DeLee and Charnley (1976). The oblique views of the acetabulum were also evaluated using three zones. Regions of the acetabular surface with radiographic evidence of noncontact on the immediate postoperative film were classified as showing gaps (attributed to failure to achieve intimate bone-prosthesis contact; Fig. 3) to distinguish them from radiolucencies first appearing on subsequent films (attributed to reactive changes with resorption of bone from areas which had previously had intimate contact) (Schmalzreid, Jasty and Harris 1992a; Fig. 4).

An acetabular component was considered definitely loose only if it migrated. Vertical and horizontal migrations were evaluated by measuring the distances from perpendiculars drawn from the hip centre to the bottom of each teardrop contour. Evidence of failure of the porous coating, such as bead shedding or disruption of the porous mesh, was also recorded.

Radiolucencies were recorded in $0.5 \mathrm{~mm}$ increments by zone in each of the three projections. Since retrieval
Table I. Clinical results in 96 surviving hips

\begin{tabular}{lrrr}
\hline & ARC & HGP & All hybrids \\
\hline Follow-up in months (mean) & 85 & 67 & 76 \\
Age in years (mean) & 56 & 66 & 61 \\
& & & \\
Harris hip score & 12 & 22 & 34 \\
$\quad$ Perfect (100 points) & 25 & 16 & 41 \\
$\quad$ Excellent, not perfect & 6 & 7 & 13 \\
Good & 5 & 1 & 6 \\
Fair & 2 & 0 & 2 \\
Poor & 92 & 95 & 93 \\
Mean score & & & \\
Pain score & 21 & 35 & 56 \\
$\quad$ None & 21 & 10 & 31 \\
Slight & 5 & 2 & 7 \\
Mild & 3 & 0 & 3 \\
Moderate & 39 & 43 & 41 \\
$\quad$ Mean score & & & \\
\hline
\end{tabular}

studies indicate that bone ingrowth may continue to accrue for up to two years, we arbitrarily defined the first two years as a period of interface maturation. We therefore specified a progressive radiolucency at the prosthesis-bone interface as one which first appeared or had increased in either width or extent between the twoyear and the final film.

We used the Fisher exact test and the rank-sum test to determine the statistical significance of the data.

\section{RESULTS}

The average Harris hip score at final follow-up was 93 (Table I): 75 hips were excellent, 13 good, 6 fair and 2 poor, giving $91 \%$ good or excellent results. The Harris hip score was slightly better for the HGP/Precoat group (95) compared with the ARC-hybrid (92). The number of excellent results was roughly equivalent for both types of implant, but patients with the HGP Precoat combi- 


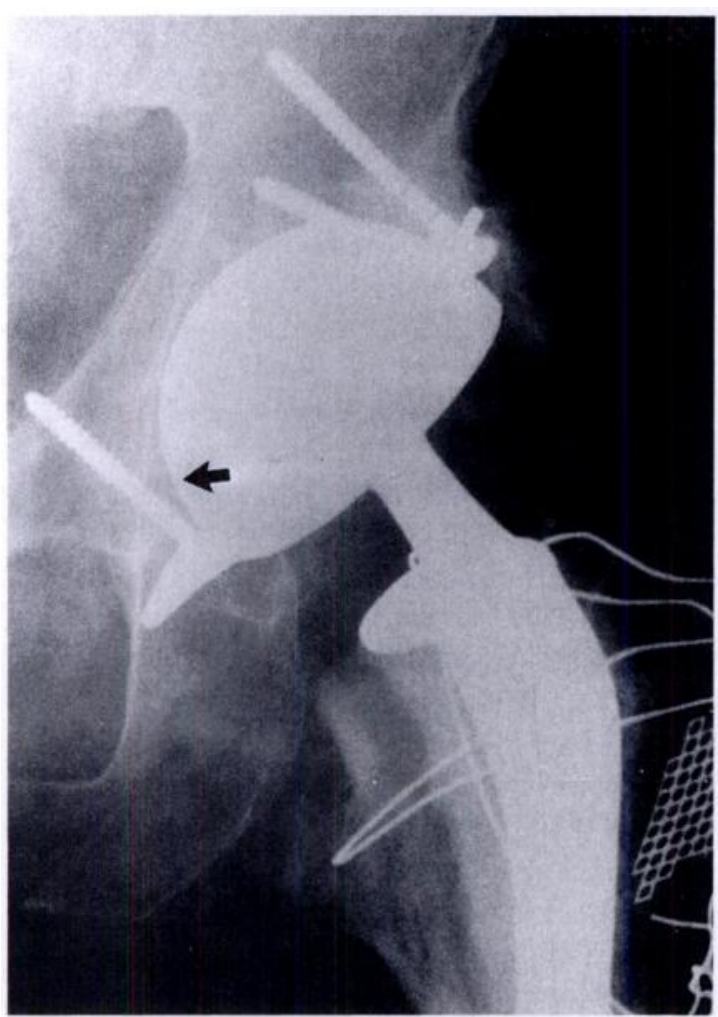

Fig. 4a

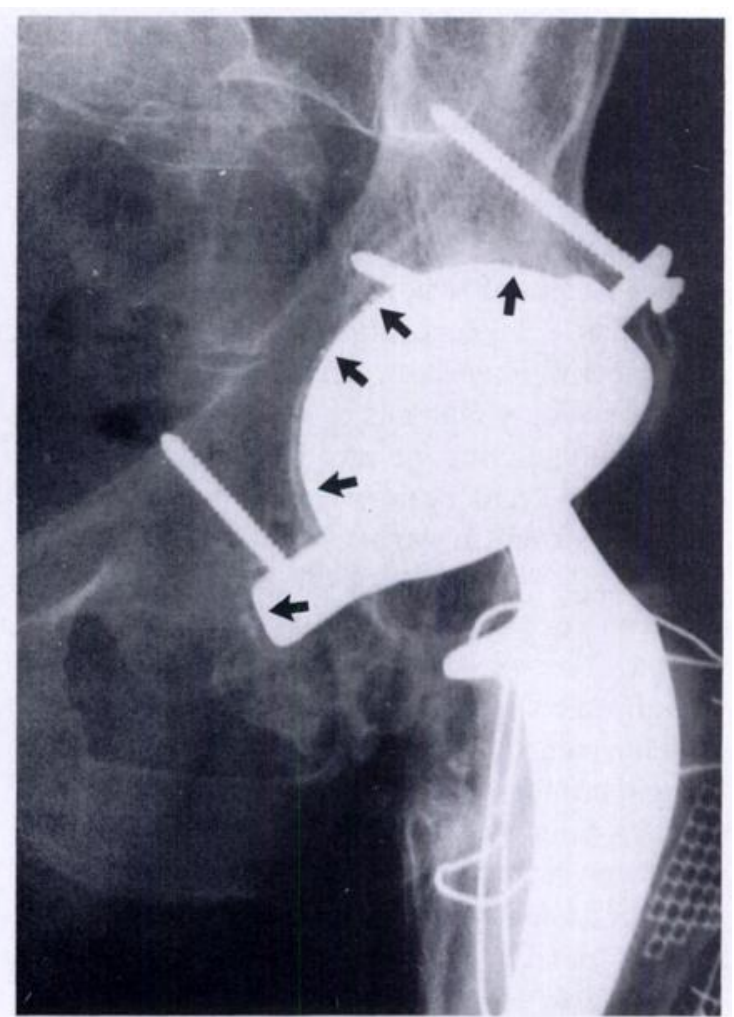

Fig. 4b

Figure 4a - Postoperative radiograph of an ARC-hybrid total hip replacement showing excellent contact between the porous surface of the acetabular component and the bone. Figure $4 \mathrm{~b}$ - At nearly eight years there is progressive radiolucency which is most obvious in zone 3 but is also present in zones 1 and 2 . There is bead loss, which is also progressive. The loss of calcar bone in this case results from surgical resection associated with the excision of Brooker grade 4 heterotopic ossification (Harris and Maloney 1989).

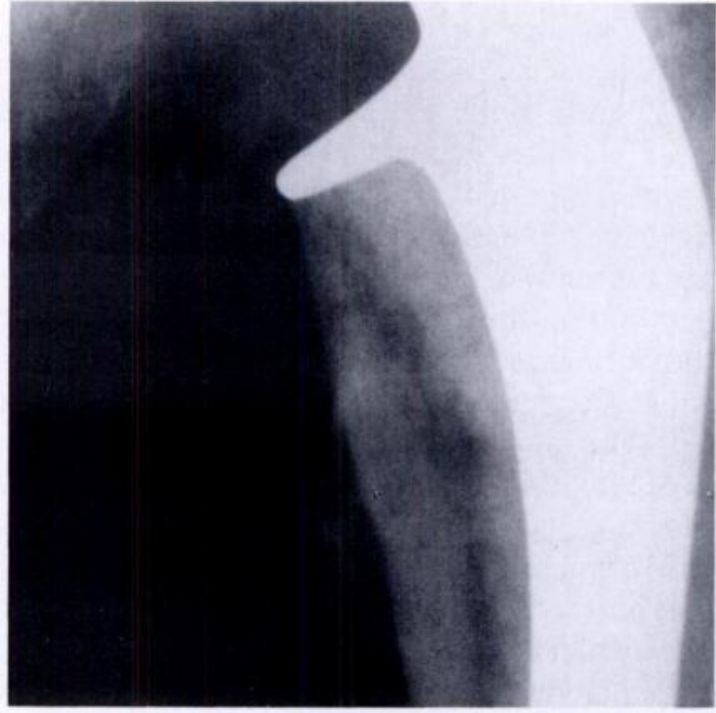

Fig. 5a

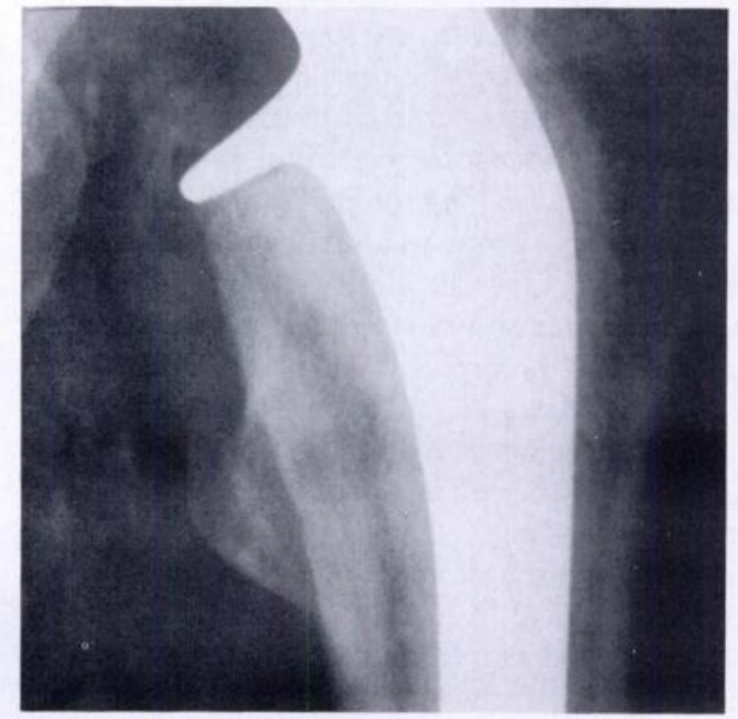

Fig. 5b

Figure 5a - Postoperative radiograph of an HD-2 hybrid total hip replacement showing excellent contact between the collar and the bone on the medial cortex. Figure $5 b$ - Five years later, showing excellent maintenance of the contact and good bone density in the medial neck. 
nation had a higher percentage $(p<0.01)$ of perfect results.

No patient had severe pain, and $88 \%$ of hips had no or only slight pain. Patients with the HGP/Precoat implant had an average pain score 4 points higher than the ARC-hybrid ( 43 to $39 ; \mathrm{p}<0.01$ ). This difference appeared to reflect the higher number of patients with the HGP/Precoat implant with no pain as against the higher number of patients with the ARC-hybrid with slight pain (Table I). Postoperative walking ability was excellent with no difference between the two groups: $92 \%$ of patients could walk six blocks or more, $84 \%$ required no aid, and $95 \%$ had no limp or only a slight limp.

In all, 79 stems had been inserted in a neutral or valgus position and ten in varus. No standard femoral stem had loosened. No HGP socket was loose and no socket had been revised for loosening. There were no infections. Four components in three hips, however, have been revised. Both components of one ARC-hybrid have been revised for recurrent dislocation, one custom $\mathrm{CDH}$ femoral component for loosening, and one bone-ingrown HGP socket for failure of the liner-locking mechanism. While these three replacements are regarded as overall failures, we are still following the unrevised HGP socket in one of them and the unrevised Precoat stem in the other.

In those hips in which the medial neck had been present at the original operation (it was absent in seven cases), there was radiographic evidence that 'collarcalcar' contact had been achieved at surgery in 75 of 82 cases $(91 \%)$. In these 75 hips, the collar-calcar contact had been maintained at final follow-up in all but nine $(88 \%$; Fig. 5). No decrease in the bone mass of the medial neck was discernible on plain radiographs in 23 of the femurs with collar-calcar contact; some decrease in calcar bone mass was apparent in the other 43 hips. Osteoporosis or thinning of the calcar occurred to some degree in 49 hips but progressive osteoporosis or thinning was observed in only four. There was longitudinal loss of calcar bone ranging from 1 to $12 \mathrm{~mm}$ in nine hips and this was progressive in six. Overall, a progressive decrease in calcar bone mass was observed in 22 hips. In addition to the four hips with progressive osteoporosis, ten other hips showed progressive changes in lesions which appeared to be scalloped or 'punched-out'. Seven had rounding-off under the collar, and one a combination of scalloping and rounding.

The radiographic grading of the cement mantle was $A$ in 26 hips, $B$ in 37, C in 26 and D in none: $71 \%$ had no radiographically detectable mantle defects. Of the 26 with grade $C$ focal defects, 18 had a void or defect which was apparent only in a single radiographic zone (see Fig. 1).

One custom $\mathrm{CDH}$ femoral component showed radiographic loosening at the prosthesis-cement interface. The patient had mild, activity-related pain, a Harris hip score of 73, and was the only patient complaining of thigh pain at the latest follow-up. No other stem showed any evidence of loosening.

New lucencies at the cement-bone interface were identified in the most proximal femoral zones in only two hips, but a focal area of endosteal cortical erosion (osteolysis: Jasty et al 1986) was identified in the lateral femur in two other cases. There was femoral hypertrophy in 20 cases, seen only in the diaphyseal cortex in zones 3 and 5, and not associated with age, sex, weight, stem type, stem size, stem position, or cement grade.

The average cup had a lateral opening of $34^{\circ}\left(25^{\circ}\right.$ to $\left.45^{\circ}\right) ; 82$ were in anteversion, five neutral and two retroverted. Coverage of the acetabular component surface by bone was over $90 \%$ in 82 of the 89 radiographed hips.

There were gaps at the implant-bone interface immediately after operation in 31 hips, but these gaps were generally less than $0.5 \mathrm{~mm}$ and never more than $1 \mathrm{~mm}$. Their location appeared to be related to technique and implant: 13 of the 16 gaps in zone 2 were around the apex of an ARC implant while, in contrast, 15 HGP sockets showed gaps at the periphery, ten in zone 1 and nine in zone 3 . Three hips showed gaps in both zones 1 and 3 and one had gaps in all three zones.

Of the 16 cases with gaps observed around ARC components, in nine they were no longer visible at two years, in two the width had decreased, and in five the gap persisted. In all nine with complete resolution of the gap, and in the two with a decreased size, the gap had been in zone 2 (Fig. 4). Of the 20 zones with gaps around 15 HGP sockets, six were not visible on the two-year film, one had decreased, and 13 had persisted.

The quality of the initial fit did not appear to affect the development of a radiolucent line on the two-year follow-up film. On the final films, there were progressive radiolucencies around seven ARC and 11 HGP sockets. In all seven of the ARC sockets, progression was in zone 3 , in two cases it extended into zone 2 , and in one there were discontinuous radiolucencies in all three zones (Fig. 4). Of the 11 HGP sockets, seven showed a progressive radiolucency in zone 1 , seven in zone 3 , and three in zone 2. On the final radiographs, six HGP sockets had discontinuous radiolucencies in all three zones. No socket showed continuous radiolucency in all three zones.

The initial fit of the ARC socket did not appear to relate to the subsequent development of progressive radiolucencies; there were five progressive radiolucencies in 31 components without gaps and two in 16 with gaps. The presence of a gap in an HGP acetabular component, however, may have prognostic significance: five progressive radiolucent lines were seen around 24 components without gaps, and six of the 15 with gaps.

Two ARC sockets had migrated; in both cases the radiolucency was limited to zone three of the interface. Progressive bead shedding occurred in 14 ARC sockets; this was seen in both components that had migrated and in five of the seven with progressive radiolucency 
(Fig. 4). None of the HGP acetabular components had measurable migration or any apparent disruption of the fibre mesh. One screw broke within one year in an ARC with no apparent consequence, and there has been no screw bending, breaking or loosening associated with the HGP acetabular component. Acetabular osteolysis was observed in association with two radiographically wellfixed ARC components. The osteolysis was confined to the ilium in one case and to the pubis in the other. No acetabular osteolysis was seen in association with the HGP components.

\section{DISCUSSION}

After an average follow-up of 6.5 years, the clinical result of the hybrid hip remains excellent. The mean Harris hip score of 93 shows no deterioration since the minimum two-year follow-up, with $90 \%$ of patients having a good or excellent result and $90 \%$ no pain or only slight pain. Only the patient with a radiographically loose femoral component complained of thigh pain.

Three hips have been revised and are considered to be failures, but component loosening was the reason for revision in only one case, and that was a custom $\mathrm{CDH}$ femoral component. The only radiographically loose femoral component was also a custom $\mathrm{CDH}$ stem.

Improved femoral cementing techniques have already shown a marked enhancement of long-term fixation of femoral components (Roberts et al 1986; Russotti et al 1988; Mulroy and Harris 1990; Barrack et al 1992). Using second-generation cement techniques, Mulroy and Harris have reported only $3 \%$ femoral loosening in an average 11-year follow-up. In several series the rate of loosening of cemented femoral components has been reported to be less in the second five years than in the first five years (Stauffer 1982; Sutherland et al 1982; Mulroy and Harris 1990), and it may be that the cohort of hybrid replacements which we now report has passed the period of highest risk for femoral component loosening.

Laboratory data have indicated that an effective transfer of load from a collar to the calcar will decrease stress shielding and reduce strains in the proximal cement (O'Connor et al 1991). This is the first series of cemented femoral components in which new and optimal instruments were used for the preparation of the medial femoral neck, such as a stable femoral rasp which has a trunnion to guide the calcar planer. These instruments allowed us to obtain good collar-medial neck contact in $91 \%$ of the hips with an adequate medial neck. Moreover, this contact was maintained in $88 \%$ over the mean follow-up period of 6.5 years. These data answer some of the questions about achievability and durability of 'collarcalcar' contact (Markolf, Amstutz and Hirschowitz 1980).

Despite the maintenance of contact in $80 \%$ of cases, some loss of calcar bone was common. The varied radiographic appearance of the bone loss suggests that the cause of this loss is multifactorial and includes bone lysis secondary to particulate wear debris as well as stress shielding (Johnston and Crowninshield 1983; Johanson et al 1986).

New lucencies at the femoral cement-bone interface were rarely seen. Recent studies of well-functioning, long-term and mechanically stable femoral components retrieved at post mortem have shown that most cementbone radiolucencies represent endosteal remodelling rather than disruption of implant fixation (Maloney et al 1989; Jasty et al 1990; Kwong et al 1991). We have therefore abandoned the use of our older classification of femoral fixation as 'possibly loose' or 'probably loose' based on the extent of radiolucency at the cement-bone interface (Harris et al 1982).

With follow-up as long as eight years, we found femoral osteolysis in only two hips $(2.2 \%)$. In both cases, the lytic lesion was a small focal area related to a defect in the cement mantle adjacent to femoral components that were not loose (Jasty et al 1986). It therefore seems that improved cementing techniques also reduce the incidence of femoral osteolysis.

No sockets were revised for loosening, showing that the porous ingrowth sockets have performed at least as well as cemented acetabular components at a similar length of follow-up (Harris and White 1982; Sutherland et al 1982; Russotti et al 1988; Ritter et al 1990). This, however, does not necessarily ensure improved long-term function; loosening of cemented acetabular components increases with time, especially after the first ten years, leading to a high number of relatively late acetabular failures (Charnley 1979; Stauffer 1982; Sutherland et al 1982; Wroblewski 1986; Johnston et al 1990; Mulroy and Harris 1990).

Two of our beaded cobalt-chrome components have migrated, but none of the HGP acetabular components did so. This compares favourably with cemented acetabular components, but longer follow-up will be required. DeLee and Charnley (1976) reported that the average time for the start of migration of cemented acetabular components was greater than five years.

The interpretation of the radiographic appearance of a cementless acetabular component with regular geometry may be substantially different from that of a cemented cup with a cement mantle of irregular geometry. The absence of interface radiolucencies does not necessarily ensure bone ingrowth, and, conversely, a cementless component with radiolucencies on one view may have 'spot welds' or areas of bone ingrowth in regions that are out of the plane of the radiograph. It is also possible that a hemispherical cementless acetabular component can migrate into an equal hemisphere of bone resorption (Engh, Griffin and Marx 1990); this was seen in the two ARC sockets that migrated in the present series. This means that the measurement of small amounts of migration is important, and, unfortunately, the accuracy of our technique for measuring migration on 
standard clinical radiographs is not better than $2 \mathrm{~mm}$ (Massin, Schmidt and Engh 1989; Nunn et al 1989). There is a clear need for a simple method to detect reliably more subtle changes in position (Baldursson et al 1980; Amstutz et al 1986).

Progressive loss of beads from porous-coated cobaltchrome implants manufactured using similar techniques has been previously reported (Callaghan, Dysart and Savory 1988; Wixson, Stulberg and Mehlhoff 1991). We saw progressive bead loss in $28 \%$ of the ARC sockets, including the two with definite acetabular loosening and five of the seven with progressive radiolucencies. These observations support the proposal that progressive bead loss is the result of motion and is an indication that the implant may be loose.

We used two quite different socket designs. The immediate postoperative radiographs of the ARC socket showed some gaps in zone 2 , which indicated incomplete seating of the component. These gaps had generally disappeared at two years, perhaps because the parallel placement of the fixation screws allows the component to settle in under loading. These small zone 2 gaps do not appear to have any prognostic significance. The HGP acetabular component commonly showed gaps at the periphery of the component in zones 1 and 3 on postoperative films. In contrast to zone 2 gaps with the ARC socket, it was rare for a peripheral gap to fill in on a subsequent film. It has been shown that there is a greater chance of progressive radiolucency around an HGP acetabular component when a peripheral gap is present initially (Schmalzried et al 1992a).

No patient had a complete radiolucent zone although seven had discontinuous radiolucencies in all three zones. We identified progressive radiolucencies around 18 components, the significance of which is unclear. With our current understanding, however, of the relationship between radiolucencies and the mechanical stability of cement sockets (Hodgkinson, Shelley and Wroblewski 1988; Schmalzried et al 1992b), it may be that the presence of extensive and/or progressive radiolucencies around a cementless acetabular component will also prove to be a poor prognostic sign for long-term fixation.

We acknowledge and thank Frederick J. Dorey, $\mathrm{PhD}$, for his assistance with the statistical analysis of our data. This study was supported by the William H. Harris Foundation.

Benefits have been received from a commercial party related directly or indirectly to the subject of this article.

\section{REFERENCES}

Amstutz HC, Ouzounian T, Graver D, et al. The grid radiograph: a simple technique for consistent high-resolution visualization of the hip. J Bone Joint Surg [ Am] 1986; 68-A :1052-6.

Anthony PP, Gie GA, Howie CR, Ling RSM. Localised endosteal bone lysis in relation to the femoral components of cemented total hip arthroplasties. J Bone Joint Surg [ Br] 1990; 72-B:971-9.

Baldursson H, Hansson LI, Otsson TH, Selvik G. Migration of the acetabular socket after total hip replacement determined by roentgen stereophotogrammetry. Acta Orthop Scand 1980; 51: $535-40$.
Barrack RL, Mulroy RD Jr, Harris WH. Improved cementing techniques and femoral component loosening in young patients with hip arthroplasties: a 12-year radiographic review. J Bone Joint Surg [ Br] 1992; 74-B:385-9.

Burke DW, Gates EI, Harris WH. Centrifugation as a method of improving tensile and fatigue properties of acrylic bone cement. $J$ Bone Joint Surg [Am] 1984; 66-A:1265-73.

Callaghan JJ, Dysart SH, Savory CG. The uncemented porous-coated anatomic total hip prosthesis: two-year result of a prospective consecutive series. J Bone Joint Surg [ Am] 1988; 70-A :337-46.

Carbsom AS, Gentz C, Linder L. Localised bone resorption in the femur in mechanical failure of cemented total hip arthroplasties. Acta Orthop Scand 1983; 54:396-402.

Chanley J. Low friction arthroplasty of the hip: theory and practice. Berlin, etc: Springer-Verlag, 1979.

Davey JR, Harris WH. A preliminary report of the use of a cementless acetabular component with a cemented femoral component. Clin Orthop $1989 ; 245: 150-5$.

DeLee JG, Charnley J. Radiological demarcation of cemented sockets in total hip replacement. Clin Orthop 1976; $121: 20-32$.

Engh CA, Grifin WL, Marx CL. Cementless acetabular components. $J$ Bone Joint Surg [Br] 1990; 72-B:53-9.

Harris WH. Traumatic arthritis of the hip after dislocation and acetabular fractures: treatment by mold arthroplasty. J Bone Joint Surg [Am] 1969; 51-A:737-55.

Harris WH, Maloney WJ. Hybrid total hip arthroplasty. Clin Orthop $1989 ; 249: 21-9$.

Harris WH, White RE Jr. Socket fixation using a metal-backed acetabular component for total hip replacement: a minimum fiveyear follow-up. J Bone Joint Surg [ Am] 1982; 64-A :745-8.

Harris WH, McCarthy JC Jr, O'Neill DA. Femoral component loosening using contemporary techniques of femoral cement fixation. J Bone Joint Surg [Am] 1982; 64-A:1063-7.

Hodgkinson JP, Shelley P, Wroblewski BM. The correlation between the roentgenographic appearance and operative findings at the bone-cement junction of the socket in Charnley low friction arthroplasties. Clin Orthop 1988; $228: 105-9$.

Jasty MJ, Floyd WE III, Schiller AL, Goldring SR, Harris WH. Localized osteolysis in stable, non-septic total hip replacement. J Bone Joint Surg [ Am] 1986; 68-A :912-9.

Jasty M, Maloney WJ, Bragdon CR, Haire T, Harris WH. Histomorphological studies of the long-term skeletal responses to well fixed cemented femoral components. J Bone Joint Surg [Am] 1990; 72A:1220-5.

Johanson NA, Callaghan JJ, Salvati EA, Merkow RL. 14-year followup study of a patient with massive calcar resorption : a case report. Clin Orthop 1986; 213:189-96.

Johnston RC, Crowninshield RD. Roentgenologic results of total hip arthroplasty: a ten-year follow-up study. Clin Orthop 1983; 181 : 92-8.

Johnston RC, Fitzgerald RH, Harris WH, et al. Clinical and radiographic evaluation of total hip replacement: a standard system of terminology for reporting results. J Bone Joint Surg [ Am] $1990 ; 72-A: 161-8$.

Judet R, Judet J, Letournel E. Fractures of the acetabulum : classification and surgical approaches for open reduction. J Bone Joint Surg [Am] 1964; 46-A :1615-46.

Kwong LM, Jasty M, Mulroy RD Jr, Maloney WJ, Harris WH. Radiolucencies in cemented femoral total hip replacements: a correlation of radiographic and histologic findings at autopsy. Proc 37th annual meeting Orthopaedic Research Society, Anaheim $1991: 222$.

Maloney WJ, Jasty M, Burke DW, et al. Biomechanical and histologic investigation of cemented total hip arthroplasties: a study of autopsy-retrieved femurs after in vivo cycling. Clin Orthop 1989; 249:129-40.

Markolf KL, Amstutz HC, Hirschowitz DL. The effect of calcar contact on femoral component micromovement: a mechanical study. $J$ Bone Joint Surg [Am] 1980; 62-A:1315-23.

Massin P, Schmidt L, Engh CA. Evaluation of cementless acetabular component migration: an experimental study. J Arthroplasty 1989 ; $4: 245-51$ 
Mulroy RD Jr, Harris WH. The effect of improved cementing techniques on component loosening in total hip replacement: an 11-year radiographic review. J Bone Joint Surg [Br] 1990; 72B:757-60.

Nunn D, Freeman MAR, Hill PF, Evans SJW. The measurement of migration of the acetabular component of hip prostheses. $J$ Bone Joint Surg [ Br] 1989; 71-B:629-31.

O'Connor DO, Burke DW, Sedlacek RC, Harris WH. What effect does a collar have on reducing proximal cement strains in a cemented femoral total hip replacement in both gait and stairclimbing? Proc 17 th annual meeting Society for Biomaterials, Scottsdale, $1991: 206$.

Oh I, Bourne RB, Harris WH. The femoral cement compactor: an improvement in cementing technique in total hip replacement. J Bone Joint Surg [ Am] 1983; 65-A :1335-8.

Ritter MA, Keating EM, Faris PM, Brugo G. Metal-backed acetabular cups in total hip arthroplasty. J Bone Joint Surg [Am] 1990; 72A :672-7.

Roberts DW, Poss R, Kelley K. Radiographic comparison of cementing techniques in total hip arthroplasty. J Arthroplasty 1986; $1: 241-7$.

Russotti GM, Coventry MB, Stauffer RN. Cemented total hip arthroplasty with contemporary techniques: a five-year minimum follow-up study. Clin Orthop 1988; 235:141-7.
Schmalzreid TP, Jasty M, Harris WH. The Harris-Galante porouscoated acetabular component with screw fixation. J Bone Joint Surg [Am] 1992a; 74-A:1130-9.

Schmalaried TP, Kwong LM, Jasty M, et al. The mechanism of loosening of cemented acetabular components in total hip arthroplasty: analysis of specimens retrieved at autopsy. Clin Orthop 1992b; $274: 60-78$

Stauffer RN. Ten-year follow-up study of total hip replacement: with particular reference to roentgenographic loosening of the components. J Bone Joint Surg [ Am] 1982; 64-A :983-90.

Sutherland CJ, Wilde AH, Borden LS, Marks KE. A ten-year follow-up of one hundred consecutive Müller curved-stem total hip-replacement arthroplasties. J Bone Joint Surg [Am] 1982; 64-A :970-82.

Wixson RL, Stulberg SD, Mehlhoff M. Total hip replacement with cemented, uncemented, and hybrid prostheses: a comparison of clinical and radiographic results at two to four years. J Bone Joint Surg [ Am] $1991 ;$ 73-A :257-70.

Wroblewski BM. 15-21-year results of the Charnley low-friction arthroplasty. Clin Orthop 1986; $211: 30-5$. 